\section{INCREASED CERVICAL STRENGTH IS ASSOCIATED WITH REDUCED HEAD IMPACT MAGNITUDE IN INTERNATIONAL BLIND FOOTBALL}

1,2Daniel Fitzpatrick, ${ }^{3}$ Peter Thompson, ${ }^{2}$ Courtney Kipps, ${ }^{1}$ Nick Webborn. ${ }^{1}$ University of Brighton, Brighton, UK; ${ }^{2}$ University College London, London, UK; ${ }^{3}$ The Football Association, Burton Upon Trent, UK

\subsection{6/bjsports-2021-IOC.295}

Background Blind Football has the highest rate of injury of any Paralympic sport. Head injuries, including concussion, represent a large proportion of these injuries. Blind athletes are less able to anticipate impacts. It has been shown in other sports that athletes with greater neck strength are better able to resist head impacts in a laboratory, and that they are less likely to sustain a concussion.

Objective To establish whether isometric neck strength influenced head impact forces in Blind Football.

Design Observational study.

Setting International Blind Football training and matches over six-months.

Patients (or Participants) England Blind Football squad (7 males, mean age 28.6y)

Interventions (or Assessment of Risk Factors) Isometric neck strength was measured once during preseason using a handheld dynamometer. Head impact data was recorded using a head mounted accelerometer and gyroscope (GForce Tracker) attached to player's mandatory blindfolds.

Main Outcome Measurements Head impact location (front, back, left or right) was recorded. Linear regression was performed to compare mean linear acceleration and rotational velocity to isometric cervical strength (flexion, extension, left lateral flexion and right lateral flexion) with movements paired to the impact direction they oppose.

Results A total of 212.5 player hours were recorded (192.5 hours in training and 20 hours in matches). Increased cervical strength was associated with reduced mean linear acceleration $\left(\mathrm{R}^{2}=0.1912, \mathrm{p}=0.020\right)$ when comparing opposing movement to respective impact location. There was no association between cervical strength and rotational velocity $\left(R^{2}=0.001, p=0.861\right)$ or between number of impacts $\left(\mathrm{R}^{2}=0.4354 \mathrm{p}=0.1068\right)$.

Conclusions Elite Blind Football players with greater cervical strength were subject to head impacts with smaller linear acceleration. This is consistent with existing research in ablebodied athletes and provides an additional factor of the live game play setting in Blind Football. Further research is required to determine if cervical strength training can reduce the risk of concussion in Blind Footballers.

\section{LIFETIME PREVALENCE AND ONE-YEAR INCIDENCE OF SPORT-RELATED CONCUSSION IN ADOLESCENTS}

1,2,3 Jocelyn McCallum, 1,2,3,4,5,6,7 Carolyn Emery, $1,2,3$ Paul H Eliason, 1,2,3,7,8 Kathryn Schneider, ${ }^{1,2,3}$ Amanda M Black. 'Sport Injury Prevention Research Centre, Faculty of Kinesiology, University of Calgary, Calgary, Canada; ${ }^{2}$ Hotchkiss Brain Institute, Cumming School of Medicine, University of Calgary, Calgary, Canada; ${ }^{3}$ Alberta Children's Hospital Research Institute, Cumming School of Medicine, University of Calgary, Calgary, Canada; ${ }^{4}$ Community Health Sciences, Cumming School of Medicine, University of Calgary, Calgary, Canada; ${ }^{5}$ Department of Pediatrics, Cumming School of Medicine, University of Calgary, Calgary, Canada; ${ }^{6}$ O'Brien Institute for Public Health, Cumming School of Medicine, University of Calgary, Calgary, Canada; 7 Sport Medicine Centre, University of Calgary, Calgary, Canada; ${ }^{8}$ Evidence Sport and Spinal Therapy, Calgary, Canada

$10.1136 /$ bjsports-2021-IOC.296
Background Understanding the burden of concussion among youth is important to quantify the effectiveness of communitybased concussion prevention strategies.

Objective To determine the lifetime prevalence and one-year incidence of sport-related concussion among male and female high school students (ages 14-19 years) in Alberta, Canada.

Design Cross-sectional survey.

Setting High schools (Alberta, Canada).

Participants High school students $(\mathrm{n}=2029$; 958 male, 1048 female, 23 identified as 'other'; ages 14-19 years) from 24 schools.

Assessment of Risk Factors High school students completed a web-based survey during class (October 2018 - March 2019). Students identified the top three sports for participation in the past year.

Main Outcome Measurements Self-reported lifetime prevalence and one-year incidence of sport-related concussion were described by sex and sport with 95\% confidence intervals (CI) adjusted for cluster by school.

Results Most high school students (1763/2029, 86.69\%) reported participating in a sport/recreational activity in the past year. Of the 1,971 students (97.14\%) answering the question about lifetime concussion history, 264/923 males [28.60\% (95\% CI, 21.97-36.31)], 227/1027 females [22.10\% (95\% CI, 17.96-26.89)], and 3/21 'other' students [14.29\% (95\% CI, 2.22-55.05)] reported one concussion or more. 131/923 males [14.19\% (95\% CI, 10.28-19.27)], 99/1027 females [9.64\% (95\% CI, 7.49-12.33)], and 1/21 'other' students [4.76\% (95\% CI, 0.46-35.28)] reported at least one concussion in the past year. For males, hockey [32/186, [Incidence Proportion(IP):17.20/100 students/year (95\% CI, 12.48-23.23)], lacrosse [4/29, IP: $13.79 / 100$ students/year (95\% CI, 5.27-31.52)], and rugby [8/61, IP: 13.11/100 students/year (95\% CI, 6.02-26.24)] had the highest rates of concussion. For females, ringette [4/21, IP: 19.05/100 students/year (95\% CI, 9.92-33.46)], rugby [9/58, IP: 15.52/100 students/year (95\% CI, 8.04-27.84)], and wrestling [3/25, IP: 12.00/100 students/year (95\% CI, 3.68-32.76)] had the highest rates.

Conclusions 1 in 4 high school students report one or more sport-related concussions in their lifetime, and 1 in 9 students reported one or more concussions in the past year. Prevention strategies aimed at reducing concussion burden are crucial.

\section{CONCUSSION KNOWLEDGE AND EXPERIENCE AMONGST FOOTBALL REFEREES IN ENGLAND}

${ }^{1,2}$ Craig Rosenbloom, ${ }^{3}$ Carmody Sean, ${ }^{4}$ Irfan Ahmed, ${ }^{1}$ Beasley lan, ${ }^{2}$ Cowie Charlotte. ${ }^{1}$ Queen Mary University of London, London, UK; ${ }^{2}$ The Football Association, Burton-on-Trent, UK; ${ }^{3}$ Chelsea Football Club, London, UK; ${ }^{4}$ Homerton University Hospital, London, UK

\subsection{6/bjsports-2021-IOC.297}

Objective Football players are at risk of sustaining sport-related concussions (SRC). In 2015 The Football Association (The FA) in England released guidelines promoting recognition of SRC and immediate removal from the sporting environment as an important strategy in reducing SRC associated morbidity. The responsibility to safeguard players with a suspected concussion includes all stakeholders in the game, and this extends to referees. To date, referees' experience and knowledge of concussion with particular reference to The FA concussion guidelines is unknown. 
Design Cross-sectional study.

Participants An online questionnaire based on the 5th Consensus Statement on Concussion in Sport (Berlin, 2016) and The FA concussion guidelines was distributed by The FA and the Professional Game Match Officials Limited (PGMOL) to their referee members (Queen Mary University of London Research Etics Committee QMREC2014/24/162)

Main Results 208 questionnaires were completed of which 34 $(16 \%)$ were from referees officiating in top two tiers of English football (Premier League and Championship). 48\% felt confident recognising SRC on pitch, $62 \%$ confident in the immediate management of concussion, and 69\% confident that their concussion knowledge was adequate to officiate games. Some concussion-related symptoms had high awareness, but seizure and aggression had lower recognition. 32\% incorrectly believed only a direct blow to the head could cause a concussion. $30 \%$ felt the final say on player removal was not that of the medical team. Low rates of concussion specific education or training were found, but high interest in future education.

Conclusions Improvement in concussion knowledge amongst football referees is required. An emphasis on educational initiatives aimed at these stakeholders could improve the health and welfare of those participating in football.

\section{PLAYING THE GAME OR GAMING THE SYSTEM: ARE US PRIVATE HIGH SCHOOL STUDENT-ATHLETES REPORTING, HIDING OR FAKING CONCUSSIONS?}

${ }^{1}$ Katherine Snedaker, ${ }^{2}$ Jason Bouton. ${ }^{1}$ PINK Concussions, Norwalk, CT, USA; ${ }^{2}$ KING School, Stamford, CT, USA

\subsection{6/bjsports-2021-IOC.298}

Background Sports- and recreation-related (SRR) activities are a major cause of concussions among adolescents. Most adolescent SRR concussion research has been conducted among public school students. As private schools are qualitatively different from public schools (e.g. location, socioeconomic status, sports played), this study explores the concussion experiences of a large group of private high school students.

Objective The purpose of this study is to describe concussion reporting and return to learn (RTL) and return to play (RTP) post-concussion in a sample of private high school students who play a sport or engage in a recreational activity. These outcomes were also stratified by gender, contact level of their primary sport, and grade.

Design We surveyed students who played sports or a recreational activity about the sports they played, and their selfreported concussion experiences between April - May, 2018. Descriptive, bivariate, and multivariate statistics are presented. Setting Ten New England private preparatory high schools. Patients (or Participants) Data was collected from 2,122 male and female students.

Interventions (or Assessment of Risk Factors) Sex, grade, contact level of primary sport played and age of first concussion. Main Outcome Measurements The main outcome of interest was percentage of students who reported/hid a concussion, and the time it the student to return to school and then to sport.

Results One-third (33.0\%) of students who reported engaging in sport-activities, self-reported experiencing a concussion in their lifetime. A higher percentage of males, students who played contact sports, and those who played multiple seasons of school sports, reported a concussion. Sex, contact level of primary sport played and age of first concussion were also significantly associated with reporting a sports-related concussion. 3\% of students reported faking concussions.

Conclusions A sizeable proportion of private preparatory high school students reported experiencing a concussion, with some students at higher risk.

\section{LOWER DYNAMIC NECK STRENGTH IS ASSOCIATED WITH HISTORY OF CONCUSSION IN VARSITY FEMALE SOCCER PLAYERS}

Theo Versteegh. Western University, London, Canada

\subsection{6/bjsports-2021-IOC.299}

Background There is mounting evidence that dynamic neck strength may play a role in protecting against concussion. It is also well established that athletes with a prior history of concussion are at higher risk than those with no prior history.

Objective To assess if there is a difference in dynamic neck strength between athletes with a self-declared history of concussion $(\mathrm{HxC})$ and athletes with no history of concussion (No-HxC). Secondly, to determine if dynamic neck strength can be used as a predictor for previous concussion history through a receiver operating characteristic curve (ROC) and hence, be used as a proxy for future concussion risk.

Design Observational cohort design

Setting Varsity level female competitive soccer players

Participants 28 athletes (average age 19.4 years, range 18-21), separated by self-declared history of concussion ( $\mathrm{HxC} n=10$ and No-HxC $\mathrm{n}=18$ )

Assessment Dynamic neck strength was calculated as the peak Rate of Force Development (RFD) in pounds-force per second $\left(\mathrm{lb}_{\mathrm{f}}{ }^{*} \mathrm{~s}^{-1}\right)$ achieved during 50 revolutions on the TopSpin360 neuromuscular neck-training device.

Results RFD for $\mathrm{HxC}$ was $3.85 \mathrm{lb}_{\mathrm{f}}{ }^{*} \mathrm{~s}^{-1}$ (95\% CI $2.53-5.17$ $\mathrm{lb}_{\mathrm{f}}{ }^{*} \mathrm{~s}^{-1}$ ) while RFD for No-HxC was $7.14 \mathrm{lb}_{\mathrm{f}}{ }^{*} \mathrm{~s}^{-1}$ (95\% CI $5.17-9.12 \mathrm{lb}_{\mathrm{f}}{ }^{*} \mathrm{~s}^{-1}$ ) Independent samples t test $\mathrm{p}=0.012$. ROC cut-off value of $4.5 \mathrm{lb}_{\mathrm{f}}{ }^{*} \mathrm{~s}^{-1}$ provides a sensitivity of $72 \%$ and specificity of $80 \%$ for detecting those with a history of concussion.

Conclusions In this pilot study of varsity female soccer athletes, those with a history of concussion demonstrate significantly lower dynamic neck strength measurements compared to teammates with no history of concussion. Knowing that $\mathrm{HxC}$ athletes are at higher risk of future concussion, the ROC cut-off value of $4.5 \mathrm{lb}_{\mathrm{f}}{ }^{*} \mathrm{~s}^{-1}$ provides a starting point for future studies using dynamic neck strength values for assessing baseline concussion risk in athletes.

\section{THE ROLE OF NECK STRENGTH IN MITIGATING SPORT RELATED CONCUSSION: A SYSTEMATIC REVIEW AND META-ANALYSIS}

${ }^{1}$ Theo Versteegh, ${ }^{2}$ Elliott Jonny, ${ }^{7}$ lan Gilchrist, ${ }^{3,8}$ Neil Heron, ${ }^{4}$ Pooler Archbold, ${ }^{5}$ Michael Webb, ${ }^{6}$ Kerry Peek. 'Western University, London, Canada; ${ }^{2}$ Ulster Hospital, Belfast, Ireland; ${ }^{3}$ Centre for Public Health, Belfast, Ireland; ${ }^{4}$ Royal Victoria Hospital, Belfast, Ireland; ${ }^{5}$ Ulster Rugby, Belfast, Ireland; ${ }^{6}$ University of Sydney, Sydney, Australia; ${ }^{7}$ Queen's University, Kingston, Canada; ${ }^{8}$ Keele University, Staffordshire, UK

10.1136/bjsports-2021-IOC.300 